\title{
Detecção de aglomerados espaciais de casos de neoplasia mamária em cães no município de Salvador, Bahia
}

\author{
Detection of spatial clusters for breast cancer in canines in the city of Salvador, Bahia
}

\author{
Júlia Morena de Miranda Leão Toríbio ${ }^{* *}{\text { João Moreira da Costa Neto }{ }^{\text {II }} \text { Maria Emília Bavia }}^{\text {III }}$ \\ Alessandra Estrela da Silva Lima II Luciana Lobato Cardim ${ }^{I}$ \\ Deborah Daniela Madureira Trabuco Carneiro ${ }^{I}$ Emanoel Ferreira Martins Filho ${ }^{I}$ \\ Lorena Gabriela Rocha Ribeiro ${ }^{\mathrm{I}}$
}

\section{RESUMO}

\begin{abstract}
Os tumores mamários espontâneos representam a neoplasia mais frequente em fêmeas caninas, correspondendo aproximadamente a $50 \%$ de todas as neoplasias. A maioria dos estudos científicos restringe-se a dados pontuais sobre a doença, sem a preocupação com sua distribuição geográfica ou mesmo com a possibilidade da geração de agregados desses eventos em uma determinada área. Levando-se em consideração a lacuna de informações na literatura, o presente trabalho teve como objetivo a criação de mapas temáticos da distribuição espacial das neoplasias mamárias em cadelas e a identificação de aglomerados de risco para a doença no município de Salvador, Bahia. Pela análise espacial de varredura, verificou-se que os casos de neoplasia mamária não estão homogeneamente distribuídos no município. Foi detectado um aglomerado primário estatisticamente significante $(P<0,001)$, abrangendo $67,3 \%$ dos casos estudados. Espera-se que estes resultados contribuam principalmente na elaboração de novos estudos, nos quais devem ser analisadas variáveis de caráter intrínseco e extrínseco ao animal para a identificação dos fatores de risco e elaboração de planos educacionais direcionados à promoção da saúde animal.
\end{abstract}

Palavras-chave: análise espacial de varredura, aglomerados, neoplasia mamária.

\section{ABSTRACT}

Spontaneous mammary tumors represent the most frequent type of cancer in canines, accounting for approximately $50 \%$ of all neoplasms. The majority of scientific papers cited in the literature are limited to non refined epidemiological data, without mentioning the trend of this disease in generating clusters in a given geographical area. In this context, this research aimed to create thematic maps of spatial distribution of mammary neoplasms in bitches and to identify disease clusters for the city of Salvador, Bahia. Trough the spatial analysis scanning, it was found that cases of breast cancer is not evenly distributed in the municipality. A significant primary cluster was detected $(P>0,001)$ covering $67.3 \%$ of the studied cases. Considering the gap in literature available in this field, it is believed that such results will become very important, especially in leading to new studies, where intrinsic and extrinsic variables regarding the animal must be taken into consideration and analyzed for factors risk identification to formulate educational plans targeting the promotion of animal welfare.

Key words: scanning spatial analysis, clusters, mammary tumor.

\section{INTRODUÇÃO}

Nos últimos anos, o estudo das interações entre homem e animal tem revelado que essas relações são componentes essenciais na vida de muitos proprietários de animais de companhia (LANE et al., 1998). Essa interação tem aumentado a preocupação com a sanidade animal, principalmente nos aspectos relacionado à prevenção, possibilitando também o diagnóstico precoce de algumas enfermidades. Tal fato tem contribuído para o desenvolvimento da oncologia

IPrograma de Pós-graduação em Ciência Animal nos Trópicos, Escola de Medicina Veterinária, Universidade Federal da Bahia (UFBA), 40170-110, Salvador, BA, Brasil. E-mail: jutoribio@yahoo.com.br. *Autor para correspondência.

IIDepartamento de Patologia e Clínicas, Faculdade de Medicina Veterinária, UFBA, Salvador, BA, Brasil.

IIIDepartamento de Medicina Veterinária Preventiva, Faculdade de Medicina Veterinária, UFBA, Salvador, BA, Brasil. 
veterinária, particularmente, para estudos do tumor mamário canino, o mais frequente nas fêmeas dessa espécie e considerado, por muitos pesquisadores, como um modelo comparativo adequado para o estudo do câncer de mama na mulher (SCHNEIDER et al., 1969; PELETEIRO, 1994; CASSALI, 2000; MEUTEN, 2002).

A maioria das pesquisas sobre o câncer de mama em cadelas tem como base a análise qualitativa e/ou quantitativa dos dados epidemiológicos isoladamente, sem o propósito de estudá-lo como um fenômeno de massa relacionado ao espaço geográfico em que se configura, onde elementos extrínsecos e intrínsecos ao animal seriam observados num único contexto. Entretanto, com o desenvolvimento das tecnologias computacionais, um novo enfoque tem sido dado à epidemiologia das doenças, permitindo o seu estudo espaço-temporal a partir da integração de um número ilimitado de informações, com seus mais variados atributos (BARCELLOS \& RAMALHO, 2002; GURGEL, 2003; BAVIA, 2006).

Dessa forma, a estatística espacial surge em oposição aos modelos estatísticos comuns nos estudos de saúde, envolvendo técnicas que permitem modelar os fenômenos cuja distribuição é afetada pela localização geográfica ou pela relação com vizinhança. Esta análise é constituída por um grupo de procedimentos interligados com o propósito de detectar aglomerados baseados no método de máxima verossimilhança (DRUCK et al., 2004; GOODCHILD \& HAINING, 2004; FOOK, 2005).

Como a agregação espacial de doenças pode ser atribuída a fatores socioeconômicos, demográficos, ambientais, genéticos e culturais, superpostos geograficamente ao padrão de ocorrência observado, os estudos de aglomerados são de extrema importância para a identificação de áreas de risco, consequentemente, para auxiliar na implantação de programas educacionais direcionados, que venham a contribuir de maneira satisfatória para saúde animal (BARCELLOS \& RAMALHO, 2002; BAVIA, 2006).

Dessa forma, este trabalho tem como objetivo a criação de mapas temáticos da distribuição espacial dos registros de neoplasia mamária em cadelas, diagnosticadas no Setor de Clínica Cirúrgica do Hospital de Medicina Veterinária da Universidade Federal da Bahia (HOSPMEV-UFBA), no período de janeiro de 2006 a abril de 2008, e a identificação de aglomerados dessa doença no município de Salvador, Bahia.

\section{MATERIAL E MÉTODOS}

A área de estudo foi a cidade de Salvador, localizada no Estado da Bahia, região Nordeste do
Brasil. Para a composição da base cartográfica, foi utilizada uma setorização adotada pelo Instituto Brasileiro de Geografia e Estatística (IBGE), a malha de Zona de Informação (ZI), totalizando 93 ZIs. Essas zonas são provenientes do agrupamento de setores censitários, utilizado em virtude da inexistência de um limite formal para a unidade de bairros na cidade.

Para a composição do banco de dados, utilizaram-se casos de cadelas portadoras de neoplasias mamárias diagnosticadas clinicamente no Setor de Clínica Cirúrgica do HOSPMEV-UFBA, no período de janeiro de 2006 a abril de 2008, e as informações referentes ao histórico clínico de cada animal, como: raça; idade; condição corporal; tipo de alimentação; administração de contraceptivos; número de mamas acometidas; tamanho do tumor; presença de ulceração e diagnóstico histopatológico do tumor.

Foram também incorporados dados socioeconômicos dos proprietários (renda e escolaridade) e a população de cadelas por ZI, estimada em $50 \%$ da população canina total, cujo cálculo baseiase em 12,5\% da população humana total/ano (BRASIL, 2000). A opção por $50 \%$ da população canina estudada tem como base a inexistência de um censo domiciliar canino reconhecido pela Secretaria Municipal de Saúde de Salvador. Os endereços dos casos diagnosticados foram georreferenciados através de Global Position System (GPS) e as coordenadas foram medidas em Sistema de Projeção UTM, datum SAD69.

Para detecção dos aglomerados de casos de neoplasia mamária canina na cidade de Salvador, foi utilizado o teste estatístico do tipo genérico, que inicialmente avalia a hipótese da inexistência de aglomerado de risco da doença na área em estudo, contra a hipótese alternativa da existência de algum aglomerado na área estudada, porém sem especificar onde poderia estar o aglomerado, sendo, portanto, improvável sua ocorrência ao acaso (LAWSON \& KULLDORFF, 1999; ASSUNÇÃO, 2001; PELLEGRINI, 2002).

Para execução do teste estatístico de varredura espacial, adotou-se o software SaTScan ${ }^{\mathrm{TM}}$ versão 8.0, idealizado por KULLDORFF \& NAGARWALLA (1995). Foram selecionadas as variáveis população canina por ZI/ano, número de casos por ZI/ano e as coordenadas dos centróides das ZIs. O SaTScan foi configurado para realizar a análise puramente espacial (quando os casos ocorrem em uma mesma região ou espaço); utilizar o intervalo de tempo anual; obedecer ao modelo de distribuição de Poisson (no qual a distribuição é heterogênea e os eventos são raros em relação à população); considerar aglomerado com $50 \%$ da população canina sob risco de adoecer 
(quando as taxas ou a prevalência da doença são baixas ou desconhecidas) e submeter à análise a replicação de Monte Carlo de 999 vezes.

\section{RESULTADOS}

No período de estudo, foram diagnosticadas clinicamente 336 cadelas portadoras de tumores mamários, do total de 2.204 animais atendidos. Dentre os animais acompanhados, 132 foram submetidos à mastectomia, sendo a cadeia mamária encaminhada imediatamente para a análise anatomo-histopatológica. Com base nas características desses animais, foi observado que, dentre as raças mais acometidas, estavam o Poodle, Sem Raça Definida (SRD) e o Pastor Alemão. A faixa etária predominante foi de oito a doze anos.

A distribuição espacial dos casos de neoplasia no município de Salvador encontra-se na figura 1. A análise espacial de varredura detectou um aglomerado de casos de neoplasia mamária estatisticamente significante $(\mathrm{P}<0,001)$, do tipo primário, com raio de abrangência de $5,7 \mathrm{~km}$. Esse aglomerado englobou 42 ZIs na região sudoeste da cidade com população canina estimada de 56.729 cadelas, risco relativo de 4,4 e proporção anual de casos de 132,8/100.000 animais. Foram registrados neste aglomerado 226 casos de neoplasia mamária, esperando-se, sob a hipótese nula, aproximadamente 106 casos da doença, numa relação entre observados e esperados de 2,1 (Figura 2).

Ao analisar as ZIs pertencentes ao aglomerado, verificou-se que o risco relativo da doença variou de 0,0 a 6,9 (Figura 3). Quanto às características socioeconômicas, foi observado que $87,3 \%$ da população residente na área geográfica englobada pelo aglomerado é alfabetizada; $22,2 \%$ têm renda entre 0 e 1 salário mínimo; 22,6\% têm renda entre 1 e 3 salários mínimos; $28,8 \%$ têm renda entre 3 e 10 salários mínimos e 25,0\% apresentam renda maior que 10 salários mínimos.

Com relação às características dos animais atendidos, observou-se que $63,3 \%$ das cadelas apresentavam condição corporal normal; 59,3\% eram alimentadas com acréscimo de comida caseira; $9,3 \%$ eram medicadas com contraceptivos; $28,3 \%$ apresentavam mais de cinco mamas acometidas; $42,9 \%$

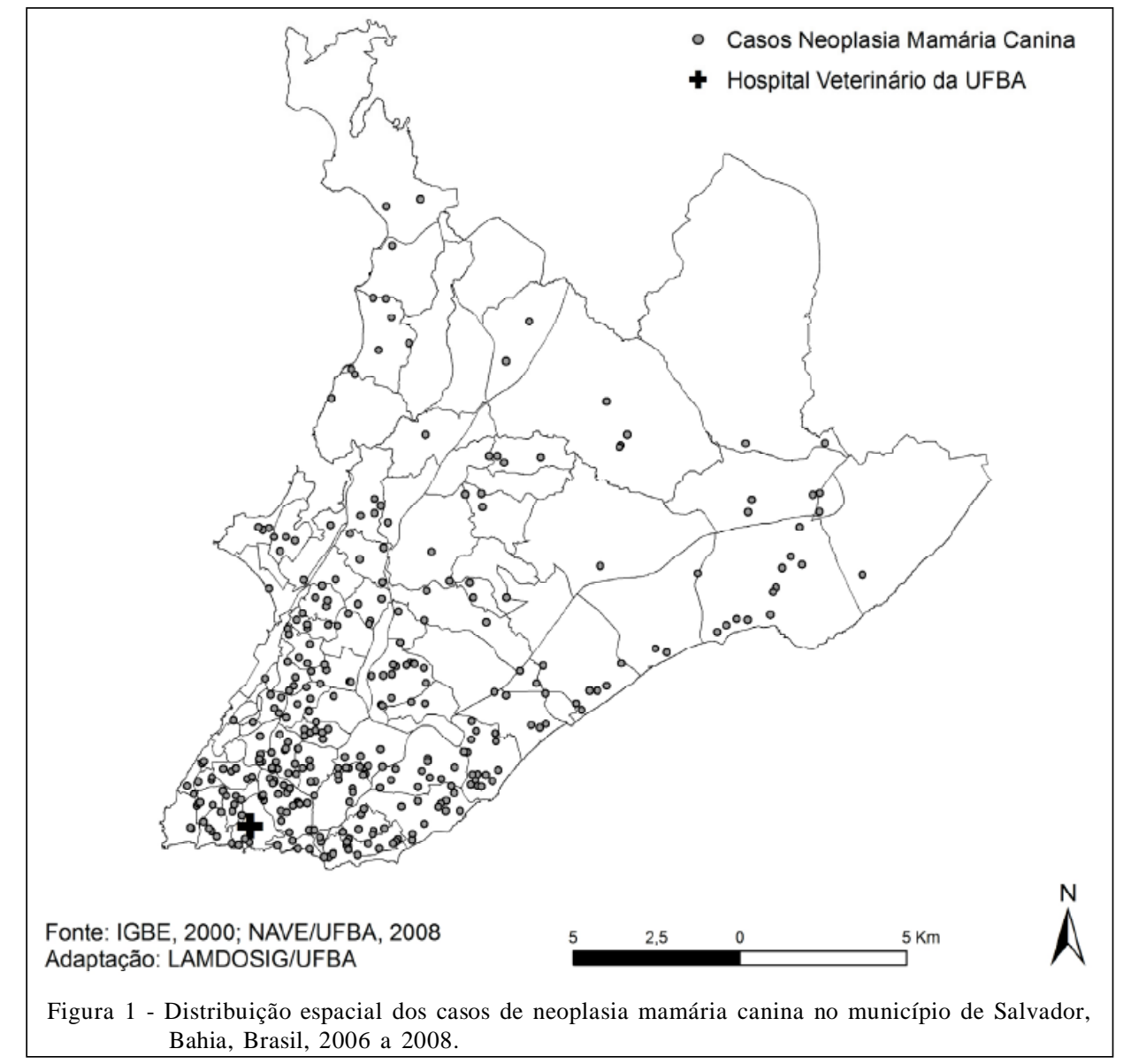

Ciência Rural, v.42, n.1, jan, 2012. 


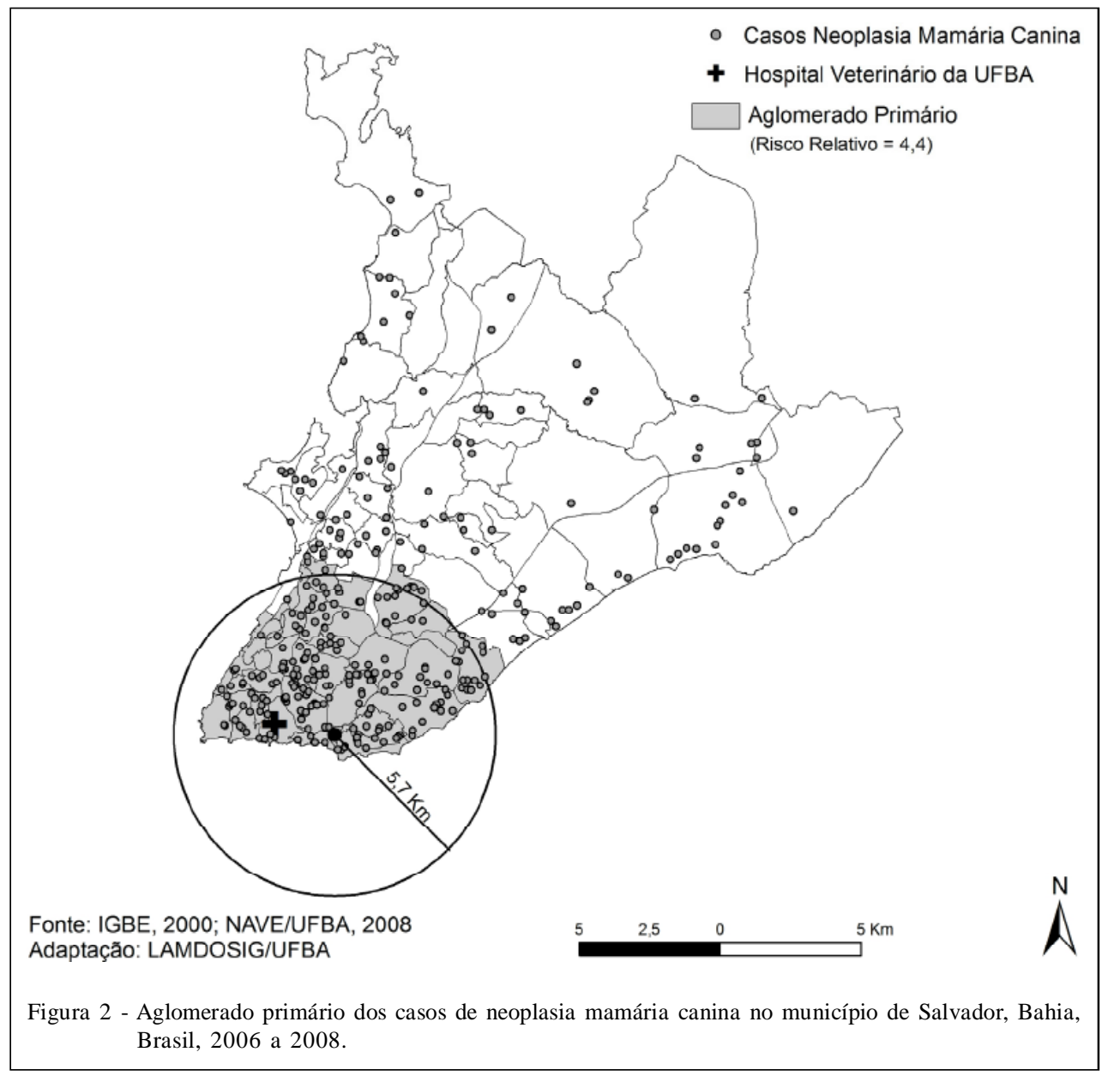

com tumores maiores que cinco centímetros; $22,1 \%$ com ulceração; 39,8\% dos tumores diagnosticados apresentavam caráter maligno e $55,8 \%$ não retornaram ao atendimento para realização da mastectomia, consequentemente não possuíam diagnóstico histológico.

\section{DISCUSSÃO}

A alta prevalência das neoplasias observadas nos animais de companhia está intimamente relacionada com a maior longevidade e maior frequência de diagnósticos (PELETEIRO, 1994; MORRISON, 1998; WITHROW, 2001; DENARDI et al., 2002; DAGLI, 2008). Os achados relativos à idade dos animais corroboram a maioria dos estudos que indicam uma maior susceptibilidade entre nove e 11 anos, com rara ocorrência de neoplasia mamária em cães com idade inferior a dois anos (BENJAMIN et al., 1999; RUTTEMAN et al., 2001; CASSALI, 2002; DE NARDI et al., 2002; OLIVEIRA et al., 2003; PIRES et al., 2003;
CAVALCANTI, 2006; FURIAN et al., 2007). Mulheres portadoras de câncer de mama com idade inferior a 30 anos e superior a 60 anos apresentam prognóstico desfavorável (HOST \& LUND, 1986), todavia, ainda não foram demonstrados, na medicina veterinária, estudos que apontem diferenças consistentes no prognóstico dos animais com base na idade (PHILIBERT et al., 2003).

PELETEIRO (1994), CASSALI (2002) e CAVALCANTI (2006) afirmam não haver predisposição racial, ainda que os tumores de mama sejam mais frequentemente diagnosticados em algumas raças. Entretanto, MEUTEN (2002) relaciona Poodle como uma das raças que apresenta predisposição genética para o desenvolvimento de neoplasias mamárias. Aparentemente, a alta incidência de Poodles com neoplasias mamárias pode estar associada ao maior número de animais com esse padrão racial ou a existência de várias linhagens e cruzamentos. Da mesma forma, a elevada incidência em cadelas sem raça definida (SRD) pode estar associada ao fato de esses animais 


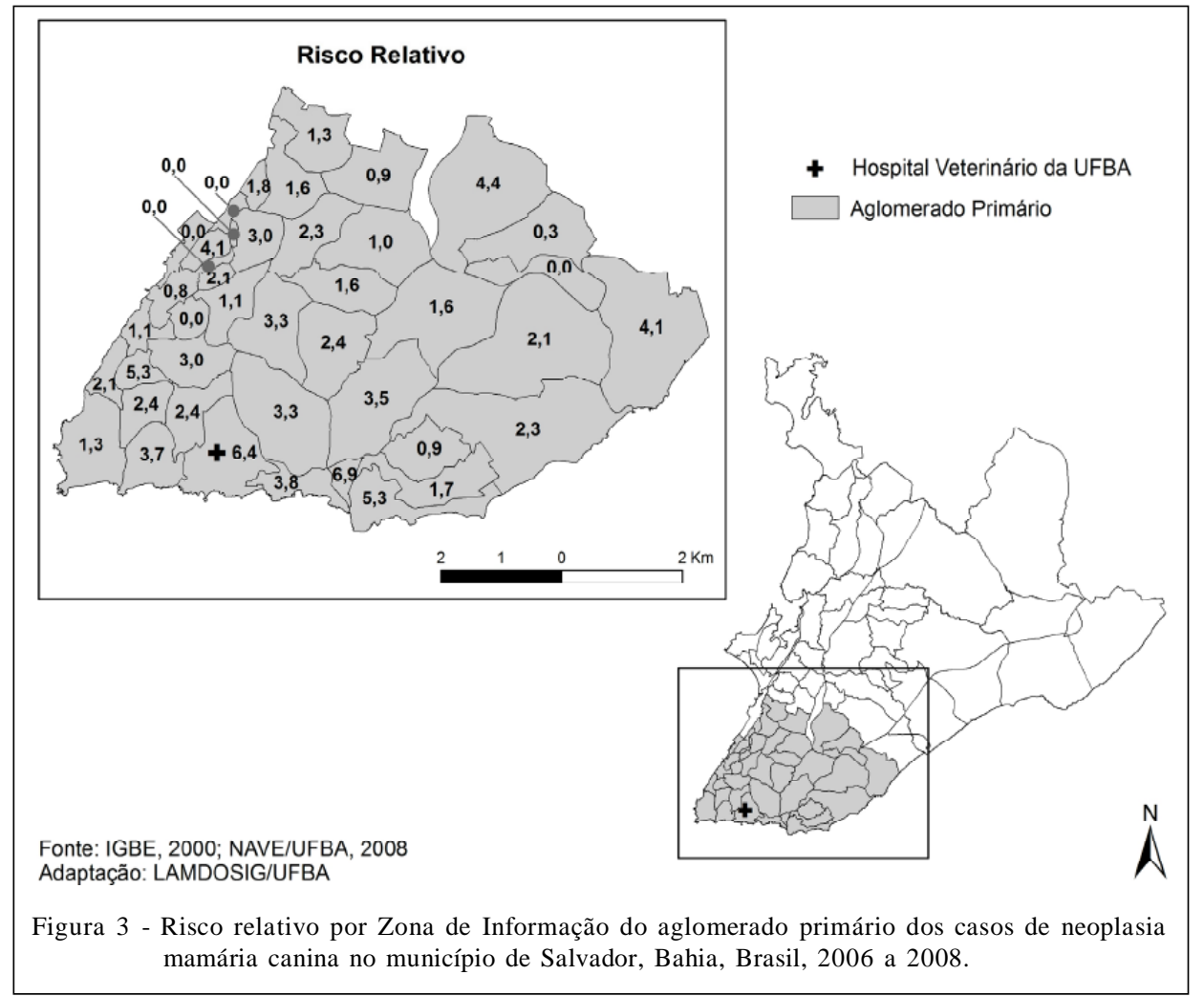

representarem a maior parte da população canina no Brasil (FONSECA, 1999; HATAKA, 2004). No campo de ação da saúde, o Sistema de Informações Geográficas (SIG), associado a testes estatísticos focados, como a análise espacial de varredura, tem sido utilizado frequentemente, pois permite observar a distribuição geográfica da doença no decorrer do tempo, seu deslocamento, índices de morbidade e modelagem de risco através da manipulação do banco de dados epidemiológico (LAWSON \& KULLDORFF, 1999; ASSUNÇÃO, 2001).

Observa-se que as Zonas de Informações pertencentes ao aglomerado primário, circunscrevem, de certa forma, áreas próximas ou contíguas ao HOSPMEV-UFBA, com distâncias de 5,7km a partir do centróide do referido aglomerado, o que, de certo modo, facilita o acesso ao serviço. Esse raio corrobora estudos internos não publicados, que indicam que a maioria dos proprietários procura o serviço Médico Veterinário com a distância máxima de cinco quilômetros de suas residências. Algumas ZIs que foram inseridas no aglomerado primário, não apresentaram casos da patologia, porém foram consideradas na análise, em virtude do princípio de TOBLER (1979) o qual diz que "todas as coisas se parecem mais próximas se parecem mais que coisas mais distantes". Outros aglomerados foram encontrados, mas não entraram na faixa de significância de 5\%, estipulada pelo método de replicação de Monte Carlo, tendo, portanto, a probabilidade de ocorrência atribuída ao acaso.

Similarmente à espécie humana, fatores socioeconômicos, atualmente ainda pouco estudados em medicina veterinária, interferem no diagnóstico do tumor mamário e no prognóstico da doença (SCHNEIDER et al., 1969; PELETEIRO, 1994; CASSALI, 2000; MEUTEN, 2002). Apesar do grande número de casos registrados no aglomerado primário, 67,3\% (226/ 336), acredita-se que esse percentual seja ainda mais elevado, pois nem todos os proprietários, por razões diversas, levam seus animais para o atendimento médico-veterinário, o que, na maioria das vezes, determina um prognóstico reservado a ruim ao paciente. Até o momento, o diagnóstico precoce do câncer de mama tem se mostrado a principal ferramenta disponível em escala populacional para o combate a essa doença, conseguindo alterar favoravelmente sua história natural. Além disso, um diagnóstico em fase inicial pode reduzir a mortalidade em consequência do câncer, possibilitando tratamentos menos agressivos (KARAYANNOPOULOU et al., 1990; SCLOWITZ et al., 2005, ESTRELA-LIMA et al., 2010). 
As características socioeconômicas observadas na área geográfica do aglomerado primário foram muito semelhantes, no entanto, é importante ressaltar que a capital soteropolitana possui características bem heterogêneas, sendo possível encontrar numa mesma ZI grande discrepância demográfica e socioeconômica entre a população residente, o que pode levar a um viés nos resultados.

A escassez de registros de neoplasia mamária em clínicas veterinárias da capital e a seleção dos indivíduos conforme demanda espontânea de atendimento no HOSPEMEV-UFBA foram as principais limitações encontradas neste estudo. Porém, os dados obtidos trazem importantes informações e servem como ponto de partida para trabalhos posteriores mais abrangentes, em que características extrínsecas e intrínsecas aos animais poderão ser contempladas para identificação de fatores de risco para a doença em espaço geográfico definido.

A maioria das cadelas avaliadas apresenta condição corporal dentro da normalidade, sugerindo pouca influência dessa variável no desenvolvimento tumoral. Entretanto, a comida caseira acrescentada na dieta pode ter propiciado a evolução dessas neoplasias, corroborando estudos de alguns autores que relatam a existência de correlação positiva entre a carcinogênese mamaria em cadelas e a alimentação caseira, principalmente elevada ingestão de carnes bovinas, suínas e gordura animal (PELETEIRO, 1994; PEREZ ALENZA et al., 1997; SORENMO et al., 2000; MOORE, 2006). O Sistema de Informações Geográficas associado à análise espacial de varredura possibilitou a identificação dos aglomerados de casos de neoplasia mamária em cadelas atendidas pelo HOSPMEV-UFBA, permitindo, através da construção de mapas temáticos, a visualização dessa distribuição espacial, o que já se posiciona como um norteador para a formulação de planos educacionais direcionados à promoção da saúde animal.

\section{CONCLUSÃO}

A distribuição espacial das neoplasias mamárias em cadelas atendidas no HOSPMEV-UFBA, no período de 2006 a 2008, mostra a formação de aglomerados de maior verossimilhança em $67,3 \%$ dos animais examinados num raio de abrangência de $5,7 \mathrm{~km}$ da localização do hospital.

\section{REFERÊNCIAS}

ASSUNÇÃO, R.M. Estatística espacial com aplicações em epidemiologia, economia e sociologia. São Carlos: Associação Brasileira de Estatística, 2001. 131p.
BARCELlOS, C.; RAMALHO, W. Situação atual do geoprocessamento e da análise de dados espaciais em saúde no Brasil. Informática Pública, v.4, n.2, p.221-230, 2002. Disponível em: <http://www.ip.pbh.gov.br/ANO4_N2_PDF/ ip0402barcellos.pdf>. Acesso em: 14 ago. 2011.

BAVIA, M.E. O controle das doenças: Trilhando os caminhos das geotecnologias. In: CHAVES, J.M.; ROCHA, W.J.S. Geotecnologias: trilhando novos caminhos nas geociências. Salvador, Sociedade Brasileira de Geotecnologia SBG- Núcleo BA/SE, 2006. p.85-98.

BENJAMIN, S.A. et al. Classifiction and behavior of canine mammary epithelial neoplasms based on life-span observations in Beagles. Veterinary Pathology, v.36, p.423-436, 1999. Disponível em: <http://vet.sagepub.com/content/36/5/ 423.full.pdf $>$. Acesso em: 14 ago. 2011. doi: 10.1354/vp.365-423.

BRASIL. Instituto Brasileiro de Geografia e Estatística. Sinopse Preliminar do Censo Demográfico, Rio de Janeiro, v.7, 415p, 2000. Disponível em: <http://www.ibge.gov.br/home/ estatistica/populacao/sinopse_preliminar/ Censo2000sinopse.pdf $>$. Acesso em: 14 ago. 2011.

CASSALI, G.D. Estudo morfológico, imunohistoquímico e citométrico de tumores mamários da cadela - aspectos comparativos com neoplasias da mama humana. 2000. 80f. Tese (Doutorado em Ciência Animal) - Escola de Veterinária, Universidade Federal de Minas Gerais, MG.

CASSALI, G.D. Patologias da glândula mamária. In: NASCIMENTO, E.F.; SANTOS, R.L. Patologia da reprodução dos animais domésticos. Rio de Janeiro, Guanabara Koogan, 2002. V.2, p.119-133.

CAVALCANTI, M.F. Fatores prognósticos na abordagem clínica e histopatológica dos carcinomas mamários de cadelas: estadiamento TNM e sistema de Nottingham. 2006. 105f. Dissertação (Mestrado em Clínica Veterinária) Faculdade de Medicina Veterinária, Universidade Federal de Minas Gerais, Belo Horizonte, MG.

DAGLI, M.L.Z. The search for suitable prognostic markers for canine mammary tumors: a promising outlook. Veterinay Journal, v.177, p.3-5, 2008. Disponível em: <http:// pubget.com/paper/18054505>. Acesso em: 14 ago. 2011. doi: $10.1016 / j . t v j 1.2007 .10 .015$

DE NARDI, A.B. et al. Prevalênica de neoplasias e modalidade de tratamentos em cães, atendidos no Hospital Veterinário da Universidade Federal do Paraná. Archives of Veterinary Science, v.7, n.2, p.15-26, 2002. Disponível em: <http:// ojs.c3sl.ufpr.br/ojs2/index.php/veterinary/article/view/3977/ 3217>. Acesso em: 14 ago. 2011.

DRUCK, S. et al. Análise espacial de dados geográficos. Brasília: EMBRAPA, 2004. 209p.

ESTRELA LIMA, A. et al. Immunophenotypic features of tumor infiltrating lymphocytes from mammary carcinomas in female dogs associated with prognostic factors and survival rates. BMC Cancer, v.10, p.256, 2010. Disponível em: <http://www.biomedcentral.com/1471-2407/10/256>. Acesso em: 14 ago. 2011. doi: 1471-2407/10/256.

FONSECA, C.S. Avaliação dos níveis séricos do $\beta$-estradiol e progesterona em cadelas portadoras de neoplasias 
mamárias. 1999. 87f. Dissertação (Mestrado em Clínica Veterinária) - Universidade Estadual Paulista, Faculdade de ciências Agrárias, Jaboticabal, SP.

FOOK, K.D. Integração da estatística espacial em ambientes GIS. 2005. 41f. Monografia Qualificação (Pósgraduação em Computação Aplicada) - INPE, São José dos Campos, SP.

FURIAN, M. et al. Estudos retrospectivo dos tumores mamários em caninos e felinos atendidos no Hospital Veterinário da FAMED entre 2003 a 2007. Revista Científica Eletrônica de Medicina Veterinária, n.8, p.20-24, 2007. Disponível em: <http://www.revista.inf.br/veterinaria08/artigos/04.pdf >. Acesso em: 14 ago. 2011.

GOODCHILD, M.F.; HAINING, R.P. GIS and spatial data analysis: covering perspectives. Papers in Regional Science, v.83, p.363-385, 2004. Disponível em: <http://hdl.handle.net/ 10.1007/s10110-003-0190-y >. Acesso em: 14 ago. 2011. doi: $10.1007 / \mathrm{s} 10110-003-0190-\mathrm{y}$.

GURGEL, H.C. A utilização das geotecnologias em estudos epidemiológicos: o exemplo da relação entre malaria e o NDVI em Roraima. In: SIMPOSIO BRASILEIRO DE SENSORIAMENTO REMOTO, 9., 2003, Belo Horizonte. Anais... Belo Horizonte: INPE, 2003. p.1303-1310. Disponível em: <http://marte.dpi.inpe.br/col/ltid.inpe.br/sbsr/ 2002/11.18.14.31/doc/12_308.pdf>. Acesso em: 14 ago. 2011.

HATAKA, A. Citologia aspirativa com agulha fina e histopatologia: valor e significado para diagnóstico e prognóstico do câncer de mama em cadelas. 2004. 90f. Tese (Doutorado em Clínica Veterinária) - Universidade Estadual Paulista, Faculdade de Medicina Veterinária e Zootecnia, Botucatu, SP.

HOST, H.; LUND, E. Age as a prognostic factor in breast cancer. Cancer, v.57, n.11, p.2217-2221, 1986. Disponível em: <http:/ / onlinelibrary.wiley.com/doi/10.1002/1097 $0142(19860601) 57: 11 \% 3 \mathrm{C} 2217$ : : A I D CNCR2820571124\%3E3.0.CO;2-T/abstract>. Acesso em: 14 ago. 2011. doi: 10.1002/1097-0142(19860601)57:11<2217::AIDCNCR2820571124>3.0.CO;2-T

KARAYANNOPOULOU M. et al. Some epidemiological aspects of canine mammary tumors treatment and prognosis. European Journal of Companion Animal Practice, v.1, p.41-47, 1990 .

KULLDORFF, M.; NAGARWALLA, N. Spatial disease clusters: detection and inference. Statistics in Medicine, v.14p.799$810,1995$.

LAWSON, A.B.; KULLDORF, M. A review of cluster detection methods. In: LAWSON, A.B. et al. (Eds.). Disease mapping and risk assessment for public health. Chichester: John Wiley and Sons, 1999. p.99-110.

LANE, D.R. et al. Dogs for the disabled: benefits to recipients and welfare of the dog. Applied Animal Behaviour Science, v.59, p.49-60, 1998. Disponível em: <http://www. journals.elsevierhealth.com/periodicals/applan/article/S01681591(98)00120-8/abstract >. Acesso em: 14 ago. 2011. doi: S0168-1591(98)00120-8.

MEUTEN, R.G. Tumors in domestic animals. 4.ed. Iowa: University of California, 2002. 788p.

MOORE, A. Advances in the treatment of mammary neoplasia. In: WORLD CONGRESS WSAVA/FECAVA/CSAVA, 2006,
Prague. Proceedings... Prague, Czech Republic: WSAVA,2006. p.562-565. Disponível em: <http://www.ivis.org/proceedings/ wsava/2006/lecture20/Moore2.pdf?LA=1>. Acesso em: 14 ago. 2011 .

MORRISON, W.B. Cancer in dogs and cats: medical and surgical management. Philadelphia: Willians e Wilkins, 1998. 785p

OLIVEIRA, L.O. et al. Aspectos epidemiológicos da neoplasia mamária canina. Acta Scientiae Veterinariae, v.31, n.2, p.105-110, 2003. Disponível em: <http://hdl.handle.net/10183/ 19802>. Acesso em: 14 ago. 2011. doi: 10183/19802.

PELETEIRO, M.C. Tumores mamários na cadela e na gata. Revista Portuguesa de Ciências Veterinárias, v.89, n.509, p.10-29, 1994

PELLEGRINI, D.C.P. Análise espaço-temporal da leptospirose no Município do Rio de Janeiro (1995-1999). 2002. 49f. Dissertação (Mestrado em saúde pública) - Escola Nacional de Saúde Pública, FIOCRUZ, RJ.

PEREZ ALENZA, M.D. et al. Relation between habitual diet and canine mammary tumors in case-control study. Journal of Veterinary Internal Medicine, v.12, p.132-139, 1997. Disponível em: <http://www.ncbi.nlm.nih.gov/pubmed/ 9595373>. Acesso em: 14 ago. 2011. doi: 9595373.

PHILIBERT, J.C. et al. Influence of host factors on survival in dogs with malignant mammary gland tumors. Journal of Veterinary Internal Medicine, v.17, p.102-106, 2003. Disponível em: <http://onlinelibrary.wiley.com/doi/10.1111/ j.1939-1676.2003.tb01330.x/abstract>. Acesso em: 14 ago. 2011. doi: 10.1111/j.1939-1676.2003.tb01330.x.

PIRES, M.A. et al. Neoplasias em canídeos - Um estudo descritivo de 6 anos. Revista Portuguesa de Ciências Veterinárias, v.98, n.547, p.111-118, 2003. Disponível em: < ht t p://www.fmv.utl.pt/s pcv/PDF/pdf9_2003/ 547_111_118.pdf>. Acesso em: 14 ago. 2011.

RUTTEMAN, G.R. et al. Tumors of the mammary gland. In: WITHROW, S.J.; MacEWEN, E.G. Small animal clincal oncology. 3.ed. Philadelphia: Saunders, 2001. p.455-477.

SCHNEIDER, R. et al. Factors influencing canine mammary cancer development and post surgical survival. Journal of the National Cancer Institute, v.43, n.6, p.1249-1261, 1969. Disponível em: <http://www.ncbi.nlm.nih.gov/pubmed/ 4319248>. Acesso em: 14 ago. 2011. doi: 4319248.

SCLOWITZ, M.L. et al. Condutas na prevenção secundária do câncer de mama e fatores associados. Revista de Saúde Pública, v.39, n.3, p.340-349, 2005. Disponível em: <http:/ /www.scielosp.org/pdf/rsp/v39n3/24786.pdf>. Acesso em: 14 ago. 2011 .

SORENMO, K. et al. Effect of spaying and timing of spaying on survival of dogs with mammary carcinoma. Journal of Veterinary Internal Medicine, v.14, n.3, p.266-270, 2000. Disponível em: <http://onlinelibrary.wiley.com/doi/10.1111/ j.1939-1676.2000.tb01165.x/abstract>. Acesso em: 14 ago. 2011. doi: 10.1111/j.1939-1676.2000.tb01165.x.

TOBLER, W.R. Cellular geography. Philosophy in geography, v.20, p.379-386, 1979.

WITHROW, S.J. Why worry about cancer in pets? In: WITHROW, S.J.; MACEWEN, E.G. Small animal clinical oncology. 3.ed. Philadelphia: Saunders, 2001. p.1-3. 